\title{
ON THE THERMODYNAMIC CONSISTENCY OF EXPERIMENTALLY DETERMINED FLUID PROPERTIES
}

\author{
Enrico Pasquini ${ }^{1 *}$, Hubertus Murrenhoff ${ }^{2}$ and Katharina Schmitz ${ }^{2}$ \\ ${ }^{1}$ FLUIDON Gesellschaft für Fluidtechnik mbH, Jülicher Straße 338a, 52070 Aachen, Germany \\ ${ }^{2}$ Institute for Fluid Power Drives and Systems, RWTH Aachen University, Campus-Boulevard 30, \\ 52074 Aachen, Germany \\ * Corresponding author: Tel.: +49 241-18298620; E-mail address: enrico.pasquini@fluidon.com
}

\begin{abstract}
In the field of fluid power, accurate knowledge of fluid properties is vital for reasonable prediction of component behaviour and system performance. In general, these properties depend on the pressure and temperature levels that the respective medium is exposed to. The properties and their respective dependencies are not publicly accessible for many fluids commonly used in fluid power. If measured values - typically published in the form of mathematical fluid property models - are available at all, their quality is typically unknown. The paper aims to provide tools to objectively ascertain the quality of measured fluid properties. For this purpose, an equation is derived which establishes a relationship between the thermodynamic parameters of density, bulk modulus, heat capacity and thermal expansion coefficient. The presented equation is always satisfied by liquids as well as gases as long as they can be treated as a continuum. Based on this relationship, the degree of thermodynamic consistency of measured properties is evaluated: The less the equation is fulfilled by experimentally determined fluid properties, the more the measured values violate physical laws. The procedure of assessing the thermodynamic consistency is demonstrated by evaluating published fluid property models with the method outlined above. To aid engineers in judging which degree of thermodynamic inconsistency is acceptable, a cut-off value is suggested.
\end{abstract}

Keywords: Fluid properties, bulk modulus, thermodynamics, fluid property model

\section{INTRODUCTION}

For numerical analysis of fluid power systems, it is essential to know the properties of the pressure medium as accurately as possible. Inaccurate fluid properties may introduce considerable error in the calculation of leakage losses, natural frequencies, component heating or other individual effects. In the worst case, the function of the realised component or even system can even be impaired because of large differences between the actual fluid properties and the values used during design calculations.

\subsection{Significance of fluid properties}

The significance of the most relevant fluid properties viscosity, bulk modulus, density, speed of sound, thermal expansion coefficient and heat capacity for component or system performance is illustrated by some practical examples.

\section{Example: Pressure drop and viscous heating}

Consider the flow through a generic resistive element, e.g. a small throttle or an orifice. Depending on whether the flow within the resistive element is laminar or turbulent, the following relations between the pressure drop $\Delta p$ across the resistor and the fluid properties hold true:

- For a given flow rate and laminar flow, the pressure drop is proportional to the absolute (dynamic) viscosity of the fluid, i.e. $\Delta p \propto \eta$.

- For a given flow rate and fully developed turbulent flow, the pressure drop is proportional to the density of the fluid, i.e. $\Delta p \propto \rho$.

As can be seen, the pressure drop depends linearly on the properties of the fluid.

For a system with imposed flow rate, this fact implies that the power requirement of the pump approximately equal to the product of pressure drop and flow rate - is heavily influenced by the fluid's properties. Thus, wrong estimates for the 
fluid's viscosity or density may lead to over- or undersized pumps.

For pressure-driven flows - e.g. a flow powered by the discharge of a pressure vessel - the strong dependence of the pressure drop on the fluid properties may lead to situations where the resulting flow rate is higher or lower than desired.

Depending on whether the dissipative heating within the resistor dominates over the generally cooling effect of expansion, the fluid's temperature will increase or decrease. If heat transfer between the fluid and the resistor can be neglected, the temperature change $\mathrm{d} T$ can be calculated as follows:

$$
\mathrm{d} T=\mu_{J T} \mathrm{~d} p=-\mu_{J T} \Delta p
$$

In this equation, $\mu_{J T}$ denotes the so-called JOULE-THOMSON coefficient which only depends on fluid properties. It is calculated based on the fluid's thermal expansion coefficient, its density and its specific heat capacity at constant pressure. As can be seen from the equation above, the sign of the JOULE-THOMSON coefficient indicates whether a pressure drop across a resistor leads to an increase $\left(\mu_{J T}<0\right)$ or decrease $\left(\mu_{J T}>\right.$ 0 ) of the fluid's temperature. Depending on the temperature and pressure level, the sign of $\mu_{J T}$ can change. Thus, it is possible that calculations based on inaccurate fluid properties may indicate a cooling of the fluid when in fact its temperature increases.

\section{Example: Natural frequencies}

Through mathematical analysis, it can be shown that the natural frequency of any fluid-power element without moving parts (e.g. pipes, large volumes etc.) is proportional to the fluid's speed of sound $a$ within the respective element:

- The natural frequencies of a fluid-filled pipe of length $L$ depend on the kinematic and dynamic boundary conditions. If both pipe ends are open or both are closed, the natural frequencies for friction-free flow are given by the following expression:

$$
f=i \frac{a}{2 L} \text { with } i \in \mathbb{N}
$$

If one pipe end is closed and one pipe end is open, the natural frequencies are equal to:

$$
f=(2 i-1) \frac{a}{4 L} \text { with } i \in \mathbb{N}
$$

- A so-called Helmholtz resonator consists of a volume $V$ and a neck of length $L$ and cross- sectional area $A$. The combination of the neck's mass and the finite stiffness of the volume constitutes a simple oscillating system. By connecting the neck to a hydraulic or pneumatic system, the resonator can be used to absorb vibrations, e.g. within a pipeline. For negligible friction, the natural frequency $f$ of the resonator is given by the following expression:

$$
f=\frac{a}{2 \pi} \sqrt{\frac{V}{L A}}
$$

The linear relationship between the fluid's speed of sound and the natural frequencies of the system delivers yet another example of how inaccurate fluid properties can have a strong impact on the calculated system behaviour. If the system is operated close to one of its natural frequencies, even small changes in the speed of sound lead to drastic changes in the system's response, e.g. pressure amplitudes.

\subsection{Determination of fluid properties}

Being aware of the significance of fluid properties for system performance, the fluid-power engineer is faced with the question how fluid properties can be obtained.

The properties of gases exposed to low pressures and high temperatures (as they are often encountered in pneumatic systems) can be calculated theoretically with satisfactory accuracy by using kinetic theory of gases [1].

Though useful for gases, statistical mechanics has failed to predict the properties of liquids with acceptable precision. Hence, for practical purposes, engineers and scientists must rely on experiments to determine the desired fluid properties. For many pure substances (e.g. water), an extensive body of literature regarding the results of such experiments is available. The data is typically presented in form of so-called fluid property models. A fluid property model is a collection of equations generated by curve fitting experimental data. These equations provide the desired physical properties (e.g. density) as functions of thermodynamic state variables, usually pressure and temperature. For the special case of water, the fluid property model developed by the International Association for the Properties of Water and Steam (IAPWS) has become an industrial quasi-standard [2]. 
Such broadly peer-reviewed and trustworthy fluid property models do not exist for many pressure media commonly used in fluid power (these are usually mixtures). In rare cases, fluid properties or even fluid property models for fluids relevant to hydraulic engineering can be taken from academic publications (e.g. the $\mathrm{PhD}$ theses of WITT or DRUMM) or commercially available software packages (e.g. TILMedia Suite) [3][4] [5]. If the practising engineer finds such a source for fluid properties at all, he is often faced with the problem of assessing data of unknown quality with respect to its reliability. One necessary, although not sufficient, indicator for the quality of measured fluid properties or fluid property models is their thermodynamic consistency. Before this concept is discusses further, the thermodynamic fundamentals with respect to fluid properties are reviewed first. Additionally, the usual definition of the bulk modulus in fluid power is subjected to a critical review.

\section{THERMODYNAMIC BACKGROUND}

The only required assumption for the following derivations is that the fluid can be considered as a continuum. This is guaranteed if the mean free path of the fluid particles is significantly smaller than the smallest characteristic dimension of the problem to be analysed, e.g. the diameter of the pipe. This requirement is satisfied for virtually all fluids and geometries encountered in the field of fluid power.

\subsection{Bulk modulus}

One of the most important fluid properties is the bulk modulus $K$. The bulk modulus characterises the resistance of a substance against a volumechange induced by a change of the hydrostatic pressure. Most fluid power textbooks define the bulk modulus as follows [6][7][8]:

$K=-V \frac{\Delta p}{\Delta V}$

In this equation, $\Delta V$ refers to the volume change, $V$ to the initial volume before the change took place and $\Delta p$ to the causative pressure difference. Even though this approximation serves a good purpose for rough engineering estimations, in this paper preference is given to a more precise, differential definition based on the intensive quantities specific volume $v$ or density $\rho$ :
$K=-v \frac{\mathrm{d} p}{\mathrm{~d} v}=\rho \frac{\mathrm{d} p}{\mathrm{~d} \rho}$

Assuming ideal gas behaviour and a general polytropic process, the following relation holds true:

$p v^{n}=\frac{p}{\rho^{n}}=$ const.

By differentiating the pressure with respect to density, one obtains an expression for the bulk modulus:

$\frac{\mathrm{d} p}{\mathrm{~d} \rho}=n \frac{p}{\rho} \rightarrow K=n \cdot p$

Hence, the bulk modulus of an ideal gas undergoing a polytropic expansion or compression is directly proportional to the respective polytropic exponent $n$.

For an isothermal process, $n$ assumes a value of unity such that the bulk modulus $K$ corresponds to the static pressure $p$ which the gas is subjected to. For isentropic processes, $n$ equals the isentropic exponent $k$ which - for ideal gases - is given by the ratio $\kappa$ of specific heats at constant pressure and volume $c_{p} / c_{v}$ [9]. It can therefore be concluded that the bulk modulus of an ideal gas is $k=c_{p} / c_{v}$ times larger when compressing the fluid isentropically rather than isothermally. For diatomic gases (e.g. $\mathrm{O}_{2}$ or $\mathrm{N}_{2}$ ), the isentropic exponent $\kappa$ and hence the ratio of both bulk moduli equals $7 / 5=1.4$ which corresponds to a difference of $40 \%$ in fluid stiffness. Since these two quite different values were both determined from equation (2), it is obvious that this relationship does not provide a unique definition of the bulk modulus of ideal gases.

A similar behaviour is to be expected for nonideal gases and liquids as well. It is therefore evident that the bulk modulus of any substance must depend on the way in which the change of state, characterised by the pressure change per density change, is performed. In order to obtain a more hands-on formulation of this change of state, the thermal equation of state is resorted to.

\subsection{Thermal equation of state}

The thermal equation of state describes the relationship between density $\rho$, temperature $T$ and pressure $p$ of a certain medium. In general, any density change $\mathrm{d} \rho$ can be decomposed into contributions due to pressure change $\mathrm{d} p$ and due to temperature change $\mathrm{d} T$ : 
$\mathrm{d} \rho=\left(\frac{\partial \rho}{\partial p}\right)_{T} \mathrm{~d} p+\left(\frac{\partial \rho}{\partial T}\right)_{p} \mathrm{~d} T$

In the above equation, the partial derivative $(\partial \rho / \partial p)_{T}$ refers to the density change per pressure change at constant temperature; accordingly, the expression $(\partial \rho / \partial T)_{p}$ denotes the density change per temperature change at constant pressure. The latter differential quantity can be expressed through the (volumetric) isobaric thermal expansion coefficient $\gamma_{p}$ [1]. This spatial counterpart of the linear thermal expansion coefficient known from the analysis of solids is defined as follows:

$\gamma_{p}:=\frac{1}{v}\left(\frac{\partial v}{\partial T}\right)_{p}=-\frac{1}{\rho}\left(\frac{\partial \rho}{\partial T}\right)_{p}$

Based on the above definition and the differential thermal equation of state, the ratio $\mathrm{d} p / \mathrm{d} \rho$ required to evaluate equation (2) is obtained:

$$
\begin{aligned}
K & =\rho \frac{\mathrm{d} p}{\mathrm{~d} \rho}=\rho \frac{1-\left(\frac{\partial \rho}{\partial T}\right)_{p} \frac{\mathrm{d} T}{\mathrm{~d} \rho}}{\left(\frac{\partial \rho}{\partial p}\right)_{T}} \\
& =\rho\left(\frac{\partial \rho}{\partial p}\right)_{T}^{-1}\left(1+\rho \gamma_{p} \frac{\mathrm{d} T}{\mathrm{~d} \rho}\right)
\end{aligned}
$$

Other than the fluid properties $\rho,(\partial \rho / \partial p)_{T}$ and $\gamma_{p}$, the bulk modulus still depends on the yet undetermined ratio of temperature change per density change, i.e. the way how the compression or expansion is conducted. This implies that the bulk modulus defined by equations (1) or (2) cannot be a fluid property in the strict sense, since different bulk moduli could be observed for the same fluid if the thermodynamic boundary conditions were changed.

For arbitrary boundary conditions and fluid properties, the theoretical determination of the differential $\mathrm{d} T / \mathrm{d} \rho$ is very demanding and, in many cases, (currently) not possible. Most applications, however, require only knowledge of the bulk modulus for two particular changes of state where certain thermodynamic quantities are kept constant. For these two special cases, an exact relationship between the temperature and density change can be specified such that a theoretical determination of the respective bulk moduli is possible.

The lowest bulk modulus is to be expected if the density change occurs at constant temperature, i.e. the compression or expansion is isothermal $(\mathrm{d} T=0)$. Such an isothermal state of state can be assumed if the density change of a fluid element happens at such a low rate that all heat supplied can be dissipated to the environment of the element ("thermal reservoir"). The bulk modulus which can be observed during such a compression or expansion is therefore referred to as the isothermal bulk modulus $K_{T}$ :

$K_{T}:=\rho\left(\frac{\partial \rho}{\partial p}\right)_{T}^{-1}$

If, on the other hand, the change of state occurs so rapidly that the compressed or expanded fluid element cannot exchange heat with its surroundings and, additionally, dissipative effects do not occur, the fluid resists compression as much as possible. Because the entropy $s$ does not change during such a lossless change of state $(\mathrm{d} s=0)$, the respective bulk modulus is termed isentropic bulk modulus $K_{s}$ :

$K_{S}:=\rho\left(\frac{\partial \rho}{\partial p}\right)_{S}^{-1}=K_{T}\left[1+\rho \gamma_{p}\left(\frac{\partial T}{\partial \rho}\right)_{S}\right]$

Determining the relationship between temperature and density changes for isentropic processes requires additional relationships. These are provided by the caloric equation of state and the fundamental thermodynamic equation.

\subsection{Caloric equation of state}

The caloric equation of state establishes a relationship between the pressure, the temperature and the energy content of a substance. If the energy content is represented by the specific enthalpy $h$, the general form of the caloric equation of state reads:

$\mathrm{d} h=\left(\frac{\partial h}{\partial T}\right)_{p} \mathrm{~d} T+\left(\frac{\partial h}{\partial p}\right)_{T} \mathrm{~d} p$

The change of enthalpy with temperature at constant pressure is known as the isobaric specific heat capacity $c_{p}$

$c_{p}=\left(\frac{\partial h}{\partial T}\right)_{p}$

Note that even though this property represents the enthalpy change per temperature change at constant pressure, the property itself is generally not constant with respect to pressure.

For the pressure-induced change in enthalpy, the following relationship can be derived using the second law of thermodynamics [9]:

$\left(\frac{\partial h}{\partial p}\right)_{T}=v\left[1-\frac{T}{v}\left(\frac{\partial v}{\partial T}\right)_{p}\right]=v\left(1-T \gamma_{p}\right)$ 
By incorporating the thermal equation of state in conjunction with the definition of the isothermal bulk modulus, the pressure differential in equation (13) can be replaced by a density change:

$$
\begin{aligned}
\mathrm{d} h & =\left[c_{p}+v \gamma_{p} K_{T}\left(1-T \gamma_{p}\right)\right] \mathrm{d} T \\
& +K_{T} \frac{1-T \gamma_{p}}{\rho^{2}} \mathrm{~d} \rho
\end{aligned}
$$

If it is known how the enthalpy changes with respect to temperature and density for an isentropic process, the desired differential $(\partial T / \partial \rho)_{S}$ can be calculated from the previous equation. This information is obtained by incorporating the fundamental thermodynamic equation.

\subsection{Fundamental thermodynamic equation}

The fundamental thermodynamic equation combines the statements of the first and second law of thermodynamics and therefore introduces constraints which limit the possibilities how a thermodynamic process can happen. With the specific enthalpy $h$, its statement can be expressed as follows [1]:

$T \mathrm{~d} s=\mathrm{d} h-\frac{\mathrm{d} p}{\rho}$

Again, the pressure change $d p$ can be substituted and the following expression is obtained:

$T \mathrm{~d} s=\left(c_{p}-\frac{T \gamma_{p}^{2} K_{T}}{\rho}\right) \mathrm{d} T-\frac{T \gamma_{p} K_{T}}{\rho^{2}} \mathrm{~d} \rho$

Since for an isentropic process $\mathrm{d} s$ equals zero, the temperature change per respect to density change is given by:

$\lim _{\mathrm{d} s \rightarrow 0}\left(\frac{\mathrm{d} T}{\mathrm{~d} \rho}\right)=\left(\frac{\partial T}{\partial \rho}\right)_{s}=\frac{T \gamma_{p} K_{T}}{\rho\left(\rho c_{p}-T \gamma_{p}^{2} K_{T}\right)}$

Since the isobaric thermal expansion coefficient is virtually always positive (with a few exceptions such as liquid water in the temperature range $0{ }^{\circ} \mathrm{C}<T<4{ }^{\circ} \mathrm{C}$ at standard pressure), an isentropic increase of density is invariably associated with an increase in temperature.

By substituting the above relation into equation (9), one arrives at the following identity [3]:

$K_{S}=\frac{K_{T}}{1-\frac{T \gamma_{p}^{2} K_{T}}{\rho c_{p}}}$

Though not new, this equation is not commonly encountered in mechanical engineering literature.

The isentropic bulk modulus $K_{s}$ constitutes the upper limit of resistance that a fluid can offer to a change in density induced by a uniformly acting pressure change. Therefore, for real, frictional and lossy density changes, the following inequality applies:

$K_{T}<K<K_{S}$

The equation above is an inequality since in reality, neither isentropic nor strictly isothermal processes are possible.

The reason why the isentropic bulk modulus of a given substance is always higher than the respective isothermal bulk modulus can be vividly explained by visualising how the fluid density depends on the pressure and temperature. A plot of the density of water versus temperature and pressure is provided in Figure 1.

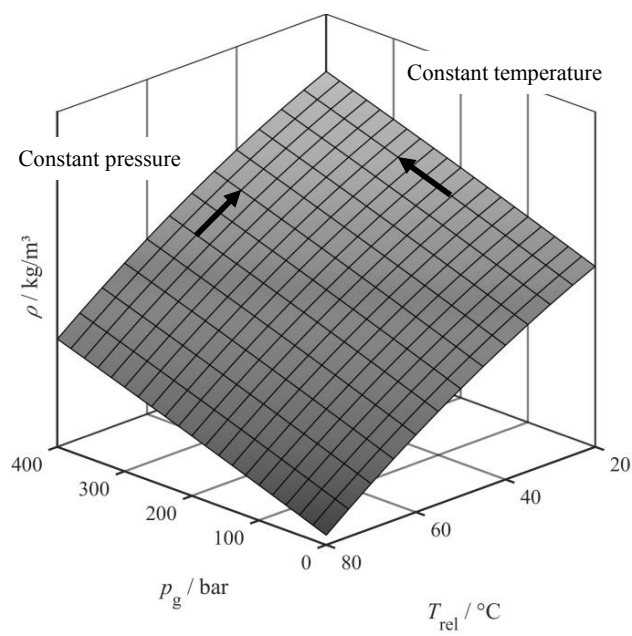

Figure 1: Density of water as a function of pressure and temperature.

As can be seen, a lower temperature (note the reversed orientation of the temperature axis) and a higher pressure generally correspond to a higher density. If the pressure is increased by $\mathrm{d} p$ at isothermal conditions, one moves along a line of constant temperature, i.e. $T=$ const. or $\mathrm{d} T=0$. Clearly, the pressure increase is associated with a higher density, i.e. $\mathrm{d} \rho>0$. If the same pressure increase $\mathrm{d} p$ were performed isentropically, the pressure rise would be associated with an increase of temperature $\mathrm{d} T>0$. The temperature change can be calculated from the following identity which is derived by combining equations (16) and definition (9): 


$$
\left(\frac{\partial T}{\partial p}\right)_{S}=\left(\frac{\partial T}{\partial \rho}\right)_{S}\left(\frac{\partial \rho}{\partial p}\right)_{S}=\frac{T \gamma_{p}}{\rho c_{p}}
$$

For water at standard pressure and a temperature of $20^{\circ} \mathrm{C}$, this expression assumes a value of approximately $1.5 \cdot 10^{-3} \mathrm{~K} / \mathrm{bar}$, i.e. a pressure increase of 1000 bar would increase the water's temperature by around $1.5 \mathrm{~K}$. Due to the increase of temperature and the subsequent (small) expansion, the gross density increase gained through increasing the pressure $\mathrm{d} p$ is reduced. Hence, for the same pressure rise, the isentropic compression results in a smaller increase of density as compared to the isothermal case, i.e. the isentropically compressed fluid is stiffer than its isothermal counterpart.

Based on the isentropic bulk modulus, the speed of sound can be calculated.

\subsection{Speed of sound}

The speed of sound indicates the speed at which pressure and velocity disturbances propagate in a fluid at rest. The mathematical definition of the speed of sound in its present-day form dates back to LAPLACE [10]. As early as 1816, LAPLACE discovered that sound waves propagate almost without losses, so that the assumption of an isentropic change of state seems permissible. Therefore, the following identity can be derived [3]:

$a_{s}:=\sqrt{\left(\frac{\partial p}{\partial \rho}\right)_{s}}$

By using definition (9), one can express the speed of sound through the isentropic bulk modulus and the density:

$a_{s}=\sqrt{\frac{K_{s}}{\rho}}$

If a bulk modulus other than the isentropic one is used for calculating the speed of sound, the propagation speed will be underestimated. When NEWTON tried to calculate the speed of sound of air in his famous principia published in 1687, he assumed an isothermal process and thus arrived at an expression which is wrong by a factor of $\sqrt{K_{S} / K_{T}} \approx 1.18$, i.e. by roughly $20 \%$ [11].

\section{THERMODYNAMIC CONSISTENCY}

With the exception of viscosity, all essential thermophysical fluid properties are linked by relation
(17). Since the equation is based on first principles and does not require the validity of any particular assumptions, the relationship applies to any fluid as long as it can be treated as a continuum.

Hence, any violation of this equation by measured fluid data or a fluid property model indicates a violation of fundamental physical laws. Here onwards, data which does not satisfy equation (17) is referred to as thermodynamically inconsistent. Due to limited accuracy of measuring devices, experimentally determined fluid properties will always be thermodynamically inconsistent to a certain degree. It is therefore important to define reasonable cut-off values above which one can actually speak of a thermodynamically inconsistent data set.

In order to obtain an indication of the degrees of consistency that can practically be achieved, published fluid property models are analysed.

\subsection{Assessment of published fluid property models (I)}

The analysis of thermodynamic consistency is demonstrated using the fluid property model presented by FLUCON GmbH [12]. The model provides equations for the calculation of all relevant thermophysical fluid properties as functions of pressure and temperature.

\section{Model equations}

Since the equations of the FLUCON model are numerical value equations, the temperature $T$ has to be provided in $\mathrm{K}$ and the gauge pressure $p_{\mathrm{g}}$ (i.e. the difference between actual pressure and atmospheric pressure) must be inserted in bar. For the density, the following model equation is proposed:

$\rho(p, T)=\frac{\rho(T)}{1-m_{1}^{\rho} \cdot \ln \left(\frac{m_{2}^{\rho}+m_{3}^{\rho} \cdot T+m_{4}^{\rho} \cdot T^{2}+m_{5}^{\rho} \cdot T^{3}+p_{\mathrm{g}}}{m_{2}^{\rho}+m_{3}^{\rho} \cdot T+m_{4}^{\rho} \cdot T^{2}+m_{5}^{\rho} \cdot T^{3}}\right)}$

In this equation, the function $\rho(T)$ refers to the temperature-dependent density at a gauge pressure of $p_{\mathrm{g}}=0$ bar and $m_{1}^{\rho} \ldots m_{5}^{\rho}$ are fluid-specific constants. The purely temperature-dependent density is given by:

$\rho(T)=\rho_{0}\left(1-T \cdot \gamma_{0}\right)$

The equation above implies that the density at a gauge pressure $p_{\mathrm{g}}=0$ bar (i.e. ambient pressure) varies linearly with temperature. Hence, the 
fluid-specific constant $\gamma_{0}$ can be interpreted as a thermal expansion coefficient, whereas the constant $\rho_{0}$ can be thought of as the density at the virtual temperature $T=0 \mathrm{~K}$.

The isentropic bulk modulus is calculated from the following equation:

$$
K_{S}(p, T)=\frac{K_{T}(p, T)}{1-\frac{\left(\rho_{0} \gamma_{0}\right)^{2} \cdot T}{\rho^{3}(p, T) \cdot c_{p}(p, T)} \cdot K_{T}(p, T)}
$$

The isothermal bulk modulus is obtained by inserting the density equation (22) into definition (8). The specific heat capacity at constant pressure $c_{p}(p, T)$ is calculated as:

$c_{p}(p, T)=c_{p}(T)-\frac{2 \cdot T \cdot \gamma_{0}^{2}}{\rho_{0}\left(1-T \cdot \gamma_{0}\right)^{3}} \cdot p_{\mathrm{g}}$

Analogous to the nomenclature used for the density, $c_{p}(T)$ denotes the purely temperature-dependent heat capacity at ambient pressure. This quantity is given by:

$c_{p}(T)=\frac{\left(\rho_{0} \cdot \gamma_{p}\right)^{2} \cdot T}{\rho^{3}(T) \cdot\left[\frac{1}{K_{T}(T)}-\frac{1}{K_{S}(T)}\right]}$

The function $K_{T}(T)$ is calculated from $K_{T}(p, T)$ for $p_{\mathrm{g}}=0 \mathrm{bar}$. The temperature-dependent isentropic bulk modulus $K_{S}(T)$ is given by:

$K_{s}(T)=\rho(T) \cdot a_{s}^{2}(T)$

The speed of sound at ambient pressure $a_{s}(T)$ is modelled through a simple polynomial expression of second order:

$a_{s}(T)=m_{1}^{a}+m_{2}^{a} \cdot T+m_{3}^{a} \cdot T^{2}$

The constants $m_{1}^{a}, m_{2}^{a}$ and $m_{3}^{a}$ are specific for each fluid. The general expression for the speed of sound is calculated based on the isentropic bulk modulus $K_{s}(p, T)$, the density $\rho(p, T)$ and the density $\rho(T)$ at zero gauge pressure:

$a_{s}(p, T)=\sqrt{K_{S}(p, T) \frac{\rho(T)}{\rho^{2}(p, T)}}$

\section{Analysis of thermodynamic consistency}

With knowledge of the equations presented in the previous section, all fluid properties required to analyse the thermodynamic consistency of the FLUCON model can be calculated. The examination is carried out for a transmission oil whose parameters were determined experimentally by FLUCON. In order to ensure that all equations and parameters were correctly transferred into the
MATLAB evaluation routine, the computed values of the individual fluid properties were compared to a reference chart provided by FLUCON.

The thermodynamic inconsistency of the FLUCON model is quantified by rearranging equation (18) such that the left-hand side equals unity:

$1=\frac{K_{S}}{K_{T}}\left(1-\frac{T \gamma_{p}^{2}}{\rho c_{p}} K_{T}\right)$

Any deviation of the right-hand side from unity indicates thermodynamic inconsistency. The relative inconsistency $\varepsilon$ can therefore be quantified by subtracting the deviant right-hand side from unity:

$\varepsilon=1-\frac{K_{S}}{K_{T}}\left(1-\frac{T \gamma_{p}^{2}}{\rho c_{p}} K_{T}\right)$

A plot for the calculated relative inconsistency $\varepsilon$ as a function of gauge pressure $p_{\mathrm{g}}$ and relative temperature $T_{\text {rel }}$ is provided in Figure 2.

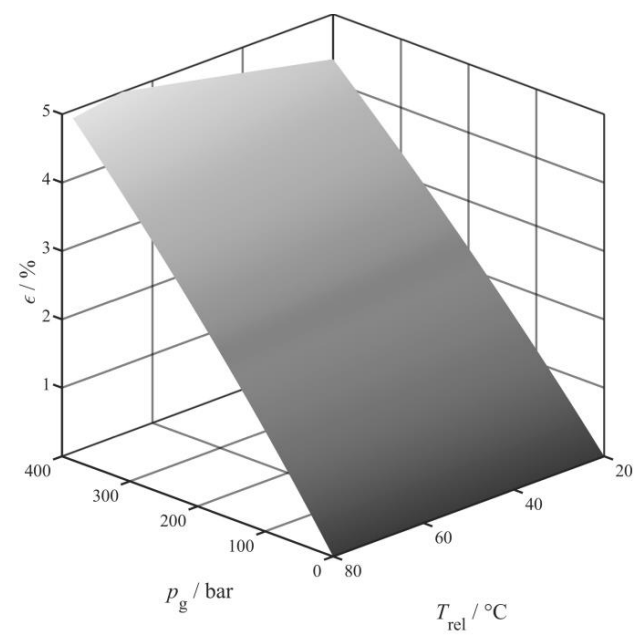

Figure 2: Thermodynamic inconsistency of the FLUCON model of a transmission oil as a function of gauge pressure and temperature.

As can be seen, the degree of inconsistency is below $5 \%$ in the covered range of pressures and temperatures. Along the isobaric line corresponding to a gauge pressure of $p_{\mathrm{g}}=0 \mathrm{bar}$, the relative inconsistency is zero for all analysed temperatures. The inconsistency increases with the pressure level at a rate of $\Delta \varepsilon / \Delta p_{g} \approx 0.0125 \% / \mathrm{bar}$. This behaviour can be explained by examining the equations constituting the FLUCON model: Even though the equations relating different fluid properties with each other appear to be physically 
sound, most of their statements are not true for pressures $p_{\mathrm{g}} \neq 0$ bar. An illustrative example of the described properties is given by equation (24). For zero gauge pressure, the statement of equation (24) is identical to the one of equation (17) since for $p_{\mathrm{g}}=0, \rho(p, T)=\rho(T)$ and hence $\gamma_{p}=$ $\gamma_{0}$. For all other pressures, this equation is invalid, since generally $\gamma_{p}=\gamma_{p}(p, T)$.

Despite these errors, the extent of thermodynamic inconsistency of the FLUCON model is more than acceptable, since the metrological limits caused by finite sensor resolutions are typically of the same order of magnitude.

\subsection{Assessment of published fluid property models (II)}

Another example is delivered by examining the fluid property model presented by DRUMM for consistency [4]. The model provides equations for the most important hydraulic fluid properties like density, viscosity, speed of sound and isentropic bulk modulus. An equation to estimate the mass-specific heat capacity is not provided. All fluid properties are modelled as functions of pressure and temperature.

\section{Model equations}

In order to model the dependency of the individual fluid properties on pressure and temperature, mostly polynomial expressions are used. Again, all model equations are numerical value equations, such that the relative temperature $T_{\text {rel }}$ has to be provided in ${ }^{\circ} \mathrm{C}$ and the gauge pressure $p_{\mathrm{g}}$ must be given in bar. The model equation for the density reads:

$$
\begin{aligned}
\rho(p, T) & =m_{1}^{\rho}+m_{2}^{\rho} \cdot T_{\text {rel }}+m_{3}^{\rho} \cdot p_{\mathrm{g}} \\
& +m_{4}^{\rho} \sqrt{p_{\mathrm{g}}+m_{5}^{\rho}}+m_{6}^{\rho} \cdot T_{\text {rel }} \cdot p_{\mathrm{g}} \\
& +m_{7}^{\rho} \cdot T_{\text {rel }}^{2}
\end{aligned}
$$

The model equation for the sound speed is given by the following expression:

$$
\begin{aligned}
a_{s}(p, T) & =m_{1}^{a}+m_{2}^{a} \cdot T_{\text {rel }}+m_{3}^{a} \cdot p_{\text {rel }} \\
& +m_{4}^{a} \sqrt{p_{\mathrm{g}}+m_{5}^{a}}+m_{6}^{a} \cdot T_{\text {rel }} \cdot p_{\mathrm{g}} \\
& +m_{7}^{a} \cdot T_{\text {rel }}^{2}
\end{aligned}
$$

Because the model presented by DRUMM does not provide a model equation for the specific heat capacity, a different approach is chosen in order to analyse its thermodynamic consistency. Since a thermal equation of state $\rho(p, T)$ as well as an equation for the speed of sound $a(p, T)$ are provided, the specific heat capacity can be calculated by rearranging equation (17):

$c_{p}(p, T)=\frac{T \gamma_{p}^{2}}{\rho\left(\frac{1}{K_{T}}-\frac{1}{K_{S}}\right)}=\frac{T\left(\frac{\partial \rho}{\partial T}\right)_{p}^{2}}{\rho^{2}\left[\frac{1}{\left(\frac{\partial p}{\partial \rho}\right)_{T}}-\frac{1}{a_{S}^{2}}\right]}$

This equation is actually used in scientific applications for determination of the specific heat capacity, particularly if direct calorimetric measurements are not possible because the pressure level of interest is too high [13].

If the coefficients $m_{i}^{\rho}$ and $m_{i}^{a}$ which DRUMM estimated for water are used to calculate values $c_{p}(p, T)$, the results can be compared to literature values for the specific heat capacity of water, taken e.g. from the commonly accepted IAPWS model [2]. Thus, even without knowledge of all quantities appearing in equation (17), the thermodynamic consistency of the DRUMM model can be assessed.

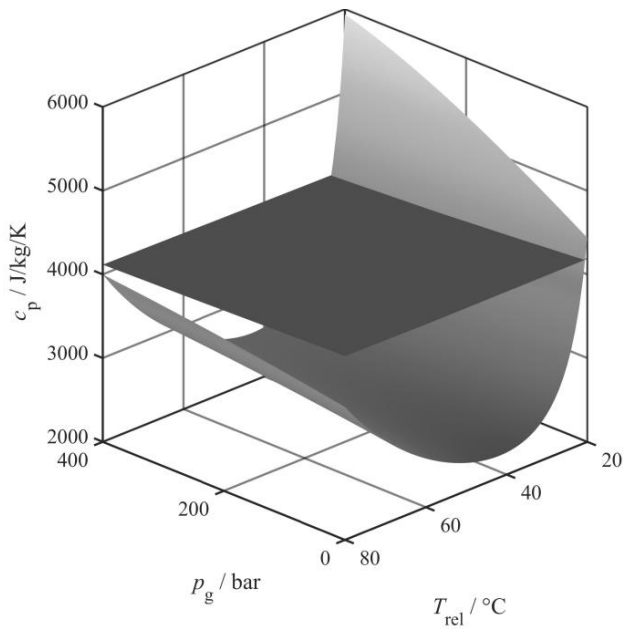

Figure 3: Calculated specific heat capacity of water as a function of pressure and temperature.

The specific heat capacity $c_{p}$ which has been calculated from the model equations of DRUMM and the corresponding parameters for water is plotted as a function of the gauge pressure and relative temperature in Figure 3 (curved surface). For comparison, the same quantity has been computed from the IAPWS model and is shown in the diagram, too (appearing as a plane) [2]. As can be seen, large differences between the DRUMM 
model and the commonly accepted IAPWS dataset exist over a wide range of temperature and pressures. The maximum and minimum values of the specific heat capacities from the IAPWS model vary by less than $4 \%$ in the analysed range, whereas the maximum $c_{p}$ calculated from the DRUMM equations exceeds the minimal value by more than $150 \%$. The calculated values of the specific heat capacity obviously contradict published and accepted values. Clearly, even without calculating the degree of thermodynamic consistency $\varepsilon$, it can be concluded that the analysed fluid property model is thermodynamically inconsistent.

The main reason for the observed behaviour of the DRUMM model can be found in the structure of the model equations themselves: Since - according to equation (35) - the slope of the density function is vital for accurate determination of the specific heat capacity, the gradients $(\partial \rho / \partial T)_{p}$ and $(\partial \rho / \partial p)_{T}$ have to be known with high accuracy. Because of the polynomial approach used, the model equations of the DRUMM model cannot properly map the curvature of the density function, even if the values of the density function might be correct.

Thus, even if the measurements on which the published model parameters are based on were carried out with perfect accuracy, a thermodynamically fluid property model could not be created with the used model equations. Hence, it is important to choose model equations of sufficient mathematical complexity which are capable of correctly mapping the relevant features of the course of the desired property as a function of pressure and temperature.

\section{SUMMARY AND OUTLOOK}

The findings of the present paper can be summarised by the following statements:

- Accurate fluid properties are vital for accurate predictions of system and component performance in the field of fluid power.

- Practising engineers are faced with the problem of ascertaining the accuracy of experimentally determined fluid properties.

- An equation was derived which establishes a connection between the most important fluid properties density, bulk modulus, thermal expansion coefficient and specific heat at constant pressure.
- Based on this identity, the thermodynamic consistency of experimentally determined fluid properties presented in the form of a fluid property model can be assessed. The more the data violates the equation, the higher the degree of thermodynamic inconsistency.

- Thermodynamic inconsistency should be below $5 \%$ to ensure accurate prediction of component or system behaviour.

- Thermodynamically inconsistent fluid properties should, if at all, be used very carefully because of the dangers outlined in the examples of chapter 1 .

- The commonly encountered, purely mechanical definition of the bulk modulus - like equation (2) - does not suffice to uniquely explicate which quantity is referred to. Depending on the thermodynamic boundary conditions during compression or expansion, the observed bulk modulus may vary significantly.

- The highest bulk modulus is encountered for an isentropic process, whereas the lowest bulk modulus is observed if the fluid's density change happens at constant temperature, i.e. if the process is isothermal.

\section{NOMENCLATURE}

$\gamma_{p} \quad$ Isobaric thermal expansion coefficient $\mathrm{K}^{-1}$

$\varepsilon \quad$ Degree of thermodyn. inconsistency \%

$\eta \quad$ Dynamic viscosity Pa.s

$\kappa \quad$ Ratio of specific heats 1

$\mu_{J T} \quad$ Joule-Thomson coefficient 1

$\rho$ Density $\quad \mathrm{kg} \cdot \mathrm{m}^{-3}$

$a_{s} \quad$ Speed of sound $\quad \mathrm{m} \cdot \mathrm{s}^{-1}$

A Cross-sectional area $\mathrm{m}^{2}$

$c_{p} \quad$ Specific heat at constant pressure $\quad \mathrm{J} \cdot \mathrm{kg}^{-1} \cdot \mathrm{K}^{-1}$

$c_{v} \quad$ Specific heat at constant volume $\quad \mathrm{J} \cdot \mathrm{kg}^{-1} \cdot \mathrm{K}^{-1}$

$f(x) \quad$ Arbitrary function of $x$

$h \quad$ Specific enthalpy $\quad \mathrm{J}^{\mathrm{kg}} \mathrm{kg}^{-1}$

$i \quad$ Integer number 1

$k \quad$ Isentropic exponent 1

$K \quad$ Bulk modulus, not specified further $\quad \mathrm{Pa}$

$K_{S} \quad$ Isentropic bulk modulus $\quad \mathrm{Pa}$

$K_{T} \quad$ Isothermal bulk modulus $\quad \mathrm{Pa}$

$L$ Length m

$\ln (x) \quad$ Natural logarithm of $x \quad 1$

$m_{i}^{x} \quad i$ th constant of the state equation for $x$

$n \quad$ Polytropic exponent 1

$p \quad$ Pressure $\quad \mathrm{Pa}$

$p_{\mathrm{g}} \quad$ Gauge pressure bar

$\Delta p \quad$ Pressure drop $\quad \mathrm{Pa}$

$s \quad$ Specific entropy $\quad \mathrm{J} \cdot \mathrm{kg}^{-1}$ 
T Temperature

$T_{\text {rel }} \quad$ Relative temperature

$v \quad$ Specific volume

$V \quad$ Volume

$\mathrm{d} f / \mathrm{d} x$ Total derivative of $f$ with respect to $x$

$\partial f / \partial x$ Partial derivative of $f$ with respect to $x$

\section{REFERENCES}

[1] BAEHR HD, KABELAC S (2016) Thermodynamik. $16^{\text {th }}$ edn. Springer Vieweg, Berlin Heidelberg

[2] IAPWS (2020) Revised Release on the IAPWS Formulation 1995 for the Thermodynamic Properties of Ordinary Water Substance for General and Scientific Use - September 2018. http://www.iapws.org/relguide/IAPWS95.html. Accessed $20^{\text {th }}$ January 2020

[3] WITT K (1974) Die Berechnung physikalischer und thermodynamischer Kennwerte von Druckflüssigkeiten, sowie die Bestimmung des Gesamtwirkungsgrades an Pumpen unter Berücksichtigung der Thermodynamik für die Druckflüssigkeit. PhD thesis, TU Eindhoven, Netherlands

[4] DRUMM SB (2012) Entwicklung von Messmethoden hydraulischer Kraftstoffeigenschaften unter Hochdruck. PhD thesis, RWTH Aachen, Germany

[5] TLK-THERMO GmbH (2020) TILMedia Suite - Softwarepaket zur Bereitstellung thermophysikalischer Stoffeigenschaften. https:// www.tlk-thermo.com/index.php/de/software/ tilmedia-suite. Accessed $20^{\text {th }}$ January 2020

[6] WILL D, GEBHARDT N (2014) Hydraulik Grundlagen, Komponenten, Systeme. $6^{\text {th }}$ edn. Springer Vieweg, Berlin Heidelberg

[7] BAUER G (2016) Ölhydraulik - Grundlagen, Bauelemente, Anwendungen. $11^{\text {th }}$ edn. Springer Vieweg, Wiesbaden

[8] WATTER H (2017) Hydraulik und Pneumatik - Grundlagen und Übungen - Anwendungen und Simulation. 5th edn. Springer Vieweg, Wiesbaden

[9] BOŠNJAKOVIĆ F, KNOCHE KF (1998) Technische Thermodynamik Teil 1. $8^{\text {th }}$, corrected edn. Steinkopff-Verlag, Heidelberg

[10] LAPLACE PS (1816) Sur la vitesse du son dans l'air et dans l'eau. Annales de chimie et de physique, t. III:297-300

[11] NEWTON I (1687) Philosophiæ naturalis principia mathematica (Mathematical principles of natural philosophy). London
[12] FLUCON FLUID CONTROL GmbH (2020) Stoffanalyse von Fluiden. https://flucon.de/dienstleistungen/stoffanalyse-von-fluiden/. Accessed $20^{\text {th }}$ January 2020

[13] GUILANO ALBO PA, LAGO S, WOLF H et al. (2017) Density, viscosity and specific heat capacity of diesel blends with rapeseed and soybean oil methyl ester. Biomass and Bioenergy, 96:87-95 